\title{
A Summary of Researches on the Construction of Experimental Teaching for Postgraduates
}

\author{
Zhong Jinhong, Han Lijun \\ School of Management, Hefei University of Technology, Hefei, China
}

\section{Email address:}

Jinhong.zhong@hfut.edu.cn (Zhong Jinhong), hanlijun198@163.com (Han Lijun)

\section{To cite this article:}

Zhong Jinhong, Han Lijun. A Summary of Researches on the Construction of Experimental Teaching for Postgraduates. Science Innovation. Vol. 8, No. 6, 2020, pp. 161-165. doi: 10.11648/j.si.20200806.11

Received: September 8, 2020; Accepted: November 10, 2020; Published: November 27, 2020

\begin{abstract}
Experimental teaching is an important part of cultivating applied talents at the postgraduate level, which plays an important role in teaching activities. In order to enhance the university's grasp of postgraduate experimental teaching, the relevant researches on postgraduate experimental teaching in China are summarized from five aspects: course-level experiment, speciality-level experiment, discipline-level or college-level public experiment, interdisciplinary innovation experiment and experimental environment construction and management. Through the analysis, it is considered that there are some deficiencies in the existing research, such as uneven results in various aspects, unbalanced distribution of discipline, and difficulty in coordination of differentiation and so on. In the future, in-depth exploration on the systematic research of postgraduat students' practical ability training, the research of public experimental teaching for postgraduates, and the research of scientific and technological experimental teaching can be carried out.
\end{abstract}

Keywords: Postgraduate Education, Experimental Teaching, Summary

\section{研究生实验教学建设研究综述}

\section{钟金宏，韩丽君}

合肥工业大学管理学院合肥, 中国

\section{邮箱}

Jinhong.zhong@hfut.edu.cn（钟金宏），hanlijun198@163.com（韩丽君）

摘要: 实验教学是培养研究生阶段应用型人才的重要环节, 在教学活动中占有重要地位。为增强各高校对于研究生实 验教学的把握, 本文从课程级实验、专业级实验、学科或学院级公共实验、跨学科创新实验以及实验环境建设与管理 五方面综述了我国对于研究生实验教学的相关研究, 分析认为现有研究存在各方面成果不均, 学科分布失衡, 差异化 难以协调等不足, 未来对于研究生实验教学的研究可从研究生实践能力培养的体系化研究, 研究生公共实验教学研究, 科技化实验教学研究等方面进行深入探索。

关键词: 研究生教育, 实验教学, 综述

\section{1. 引言}

研究生教育是培养高层次人才的主要途径, 是国家创 新体系的重要组成部分。随着经济社会发展对高层次人才 需求的增加, 以及国家对研究生教育的重视和投入增加,
我国研究生教育的规模、办学条件、办学水平和培养质量 都有了长足进步。而在当前的研究生教育中, 实践能力作 为研究生培养质量的重要指标以及研究生具备自主创新 能力的重要基础, 深受多方关注。在教育部文件《教育部、 国家发展改革委、财政部关于深化研究生教育改革的意见》 
中要求研究生培养要 “更加突出服务经济社会发展, 更加 突出创新精神和实践能力培养, 更加突出科教结合和产学 结合, 更加突出对外开放” [1]。在企业人才招聘中也对动 手能力强、更具创新意识的学生多加青睐。其中, 实验教 学活动是研究生教育中培养实践能力的重要一环。

但从研究生培养环节来看, 理论教学和学位论文环节 更为成熟和规范, 实验实践教学环节仍比较薄弱。为此, 很多高校为培养研究生实践能力, 修订研究生培养方案, 依据专家意见逐步开设研究生实验教学活动, 并不断开展 相关研究。由于实验教学与理论教学等在教学方式、环境、 内容等多方面存在较大差异, 为了帮助高校从总体上把握 我国研究生实验教学建设情况, 本文针对中国知网收录的 相关主题研究进行整理和分析, 借此更为清晰地展示现有 建设成果, 总结可能存在的不足, 并进一步探讨未来我国 研究生实验教学的建设方向, 希望给高校后续的相关教学 建设提供部分参考, 促进高校依据现有建设成果改善实验 教学情况, 形成特色的实验教学培养方案, 为社会输出更 优秀的创新实践型人才。

\section{2. 文献综述}

目前, 各高校仍在不断推进研究生实验教学活动, 随 着科技发展和知识经济兴起, 传统的研究生实验教学在教 学内容、教学方法与手段等多方面遭遇挑战。过去那些先 进的教学思想与技能在今天来看已经回归常规水平甚至 落伍, 有待进一步改革创新。范冬梅等[2]从课程内容调整、 跨专业综合实验平台建立、实验室开放与管理、师资水平、 科研氛围等方面, 给出了研究生实验教育的改革方案。后 来, 又从主体、客体、研究生实验教育管理部门等研究生 教育三元素, 探讨了研究生实验教育改革[3]。张洋等[4] 探索了新时期军校研究生实验教学的改革方法, 设想了 “学一研一战” 结合的培养模式等。但是, 暂未发现有学 者对至今以来的研究成果进行综合评述, 现有研究大多从
单方面针对某课程、专业、学科、领域或实验室环境展开, 所获研究成果亦参差不齐。

本文以 “研究生” 并 “实验” 作为主题词, 以中国知 网为检索源进行文献检索, 筛选了针对研究生实验教学的 研究成果, 通过总结已有的经验教训, 希望对未来高校研 究生实验办学起到参考作用, 并对进一步研究方向有所启 示。综述从课程级实验研究、专业级实验研究、学科或学 院级公共实验研究、跨学科创新实验与实验环境建设与管 理五个角度展开, 各类相关文献数量及分布如图1所示。

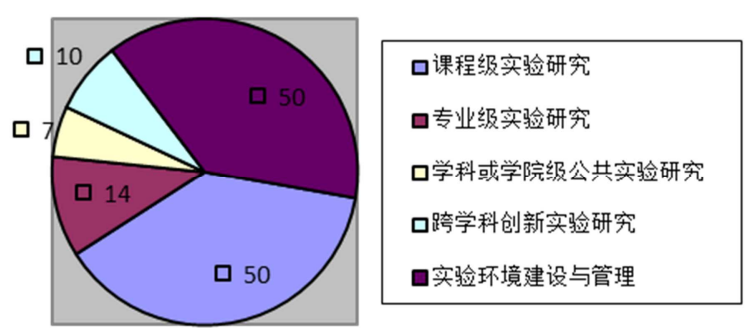

图1 相关文献数量分布。

\section{1. 课程级实验研究}

课程级实验研究主要是针对研究生课程的伴随实验 以及独立实验的课程设计。该部分研究大多具有普遍适用 性, 少数可基于学科进行发散, 整体参考价值较高。根据 课程所属学科, 列出国内对于两类研究的成果分布情况如 表1所示。其中, 课程伴随实验方面研究的关注点在课程 实验内容（体系）、教学方法、实验平台、考核评价、实 验效果评价; 而面向研究生开设的独立实验类型课程研究 主要围绕教学目标设定、实验项目选择、实验内容体系设 置与更新、教学方法与手段改进、实验条件、教学团队建 设、实验教材编写、实施方案设计、实验效率、教学效果、 考核方式、成绩评价体系等方面, 以及开展实验课程的课 程建设、教学模式、改进措施研究。

表1 课程级实验研究学科分布情况。

\begin{tabular}{|c|c|c|}
\hline \multirow{2}{*}{ 学科 } & \multicolumn{2}{|l|}{ 课程级实验研究 } \\
\hline & 课程伴随实验研究 & 独立课程实验研究 \\
\hline 管理学 & \multirow{2}{*}{$\begin{array}{l}\text { 人力资源管理、管理决策方法、量化投资 } \\
\text { 数字图像处理、信号分析与处理、线性系统理论、Petri } \\
\text { 网系统建模与仿真、VLSI设计、信息内容安全、仪器分 } \\
\text { 析及高等土力学、有机化学实验、分析仪器原理及操作 } \\
\text { 实验、扫描电子显微分析、现代分析仪器应用技术 }\end{array}$} & ERP实验 \\
\hline 工学 & & $\begin{array}{l}\text { 微型计算机系统接口技术、基于dSPACE的控制实验、自动化学科的课题研究 } \\
\text { 型实验、现代测试技术实验、光纤传感技术、近代光学实验、现代环境分析 } \\
\text { 技术、应用化学硕士生实验、传热设计性实验以及纳米力学测试 }\end{array}$ \\
\hline 医学 & 免疫学实验、医学仪器分析 & $\begin{array}{l}\text { 高级免疫学实验技术和综合性基础医学实验 } \\
\text { 生物仪器分析实验、现代微生物研究技术、现代分子生物学实验、分子生物 }\end{array}$ \\
\hline 生物学 & 细胞遗传学实验 & $\begin{array}{l}\text { 学实验、细胞生物学大实验、流式细胞术、细胞电生理、细胞培养实验、组 } \\
\text { 织培养技术、基因工程实验技术、植物生物学实验技术与动物营养学实验 }\end{array}$ \\
\hline
\end{tabular}

\section{2. 专业级实验研究}

与课程级教学研究不同, 专业级实验教学研究大多 围绕某一具体专业展开, 研究内容包括队伍建设、平台 搭建、内容设置、培养方案等多方面, 目的在于单独为 某一领域培养创新人才, 并给予高水平科学研究工作技
术一定支撑。该部分研究目前主要从教学改革和教学体 系两方面展开。

\subsection{1. 教学改革角度}

张培玉等[5]从教学体系、实验环节和课外实践3方面, 介绍了青岛大学环境工程硕士实验与实践环节的改革实 践。李彦文等[6]从实验课程学分设立、分层次实验教学内 
容设置、产学研基地创建、现代教育技术应用、实验教学 队伍建设等方面, 探索了环境专业研究生实验教学环节的 构建和实施措施。秦春节等错误!未找到引用源。[7]探讨 了基于工程实践的物流工程专业实验教学体系的改革, 把 现代物流工程环境营造到实验室, 设计了基于工程实践的 物流工程实验教学方案, 创新了物流工程实验教学方法。

\subsection{2. 教学体系角度}

娄敏和陈建民错误!未找到引用源。[8]通过设计多层次 实践培养方案, 设立综合性实验实训平台, 打造一条龙软 件实训构架, 建立顶岗式校企联合培养基地等一系列举措, 建设具有海洋石油特色的船舶与海洋工程领域专业硕士实 践教学体系。章牧等[9]从培养目标、教学内容、教学方法、 考核方式等方面, 介绍旅游管理实验教学中心的创新教学 体系。赵新建等[10]设计了旅游管理学科本科与研究生协同 发展的衔接性实验教学体系, 从交叉融合基础、专业、综 合、创新和动态等五个维度, 呈现基础认知实验、专业能 力培养、学科应用创新和产业应用研发四个阶段的发展特 征。钱猛等[11]探讨了南京农业大学“微生物学研究方法与 技术”专项培训体系的构建, 重点阐述建设内容、建设方式 及教学成效, 该培训体系分为五个功能模块, 每个模块由 专题讲座和实验技能培训构成。郑晓东等[12]通过随机分组、 教师学生共同参与的考核方式、自选课题的项目研究等方 法, 将能力和素质培养分散, 探索了光学工程研究生实验 教学新模式。程林松和李春兰[13]从实验教学培养目标、内 容设置、平台建设、实验教学模式、实验教学效果评价、 存在的问题六方面, 探讨了油气田开发工程专业研究生实 验教学模式。张英等[14]提出深化动物学专业研究生实验教 学体系构建, 通过结合简单与复杂实验、开展课内与课外 活动和加强师资力量三方面培养研究生的科研创新能力, 广泛听取研究生导师意见并时刻关注研究生相关需求。

这些研究均针对某一特殊专业进行, 研究对象明确, 但是大部分成果往往难以迁移到其他专业进行复制教学。

\section{3. 学科或学院级公共实验研究}

新世纪以来, 我国研究生生源由于招生规模扩大与来 源差异导致同一学科或专业的学生往往来自不同学校、不 同层次、不同专业, 专业水平和实践能力参差不齐, 整体 素质相对下降。为应对这一变化, 多所高校开设了学院级 或针对多个相近专业的研究生公共实验教学课程, 其特征 在于集中投入、统一管理、开放公用、资源共享，与专业 级实验教学相比包容性更强。

\subsection{1. 教学模式角度}

郑永杰等 $[15]$ 从追求卓越的研究生培养目标、大量资金 投入科研基地、研究生实验教学管理先进、采用产学研一体 化的实践教学模式等方面, 分析了日本工科研究生实践教学 经验; 指出我国应在研究生培养理念、资金投入、实践教学 管理、完善产学研结合的实践教学模式等方面加以借鉴。

\subsection{2. 教学体系角度}

刘丽琳和孙久厚 [16]从指导思想、实验项目、实验基 地、实验教师队伍、实验教材、实验教学管理模式等方面
探讨了工科研究生公共实验体系的建立。郑冬梅和王悦 [17]介绍了北京航空航天大学在研究生实验教学体系建设 上的经验: 充分发挥学科优势, 把最新科研成果融入研究 生实验教学环节; 建立跨一级学科的研究生公共实验课和 一级学科框架下的研究生专业实验课; 建立一批先进的研 究生实验教学平台; 确立前瞻性和创新性的研究生实验教 学内容; 建设高素质的研究生实验教学师资队伍。张建宇 等[18]介绍了南京理工大学化工学院在研究生公共实验教 学体系构建上的实践: 打造一流学科实验平台, 实现学科 资源共享; 依据学科实验平台建立研究生实验教学体系; 以学科实验平台的发展促进实验教学质量的提高。林小虎 等[19]探索建立以研究生创新意识和创新能力培养为导向 的农科类研究生实验教学体系, 包括搭建多专业共用的研 究生实验平台, 实现教学资源的 “统管共用”; 将农科类 专业研究生实验改造为具有综合性、设计性的探究性实验; 采用多元化的创新教学方法与模式; 改革考核方法和实验 室管理体制。赵琴等错误!未找到引用源。[20]探讨如何依 托校流体机械优势学科和流体及动力机械教育部重点实 验室, 建立科学合理的多层次研究生实验教学体系, 包括 总体思路构建、实验教学内容设置、教学模式改革, 以及 整合协同创新中心、国际研究中心等资源为教学服务。孙 腊珍等错误!未找到引用源。[21]介绍了中国科学技术大学 物理学院创新研究生实验教学体系构建上的做法, 通过开 设本硕贯通实验选修课程、研究生必修实验课程和专题实 验课程; 教学与科研相结合, 加强实验教学平台建设等方 式完善教学体系。

这些学科或学院级公共实验研究能够一定程度上加 大资源的利用率，在减少资本投入的情况下反过来增大实 验教学覆盖率并提升实践能力培养成效。

\section{4. 跨学科创新实验研究}

创新是知识经济时代的一个显著标识, 跨学科的创新 实验教学研究有助于实现高校立德树人的目标, 培养高质 量的专业人才。该部分研究成果主要包括针对特殊情形如 大型仪器设备的教学研究, 以及面向实践创新能力培养的 教学研究。

\subsection{1. 大型仪器设备角度}

由于此类设备价格昂贵、资源有限、操作复杂, 教学 模式也有别于普通实验, 该方面研究涉及其在综合实验上 的应用及作用、在院校转型中的作用、在研究生实践与创 新能力培养上的作用以及开放共享举措, 以及面向本科生、 研究生的多层次大型仪器实验教学模式、基于问题导向的 液质联用仪实验教学探究等。

\subsection{2. 创新角度}

间连山等[22]介绍了西南交通大学通过前沿实验研究 提高研究生的实际动手能力和理论水平的一些经验: 建设 一流实验室, 让学生回归实验室; 增强团队凝聚力, 提高 学生综合素质; 以实验为导向, 结合前沿研究和应用研发 让学生乐于 “享受” 研究; 以阶段性成绩鼓励学生, 进一 步提高学生自信心和积极性。杨晓华等[23]从学术生态环 境营造、科研实践训练平台搭建、创新人格培育、服务社 
会能力锻造等方面, 介绍了西安交通大学医学研究生教学 实验中心通过自主创新实验培养创新型医药专业人才的 实践经验。武卫莉等[24]从课程体系改革、授课方式改革、 学位论文形式改革、课题来源和研究内容改革等方面, 研 究材料工程领域的工程硕士创新实践能力培养模式。刘华 [25]借鉴了美国德克萨斯大学的实验教学经验, 从课程内 容、管理理念、实际操作、专题研究四方面解读其实验课 程教学模式的特点, 由此启示我国研究生创新精神及创新 能力培养。杨晓红等 [26]针对 “植物显微技术” 实验教学 进行改革, 介绍了设计性实验教学内容、方法、手段以及 教学成果, 由此探索研究生创新能力培养。孙建和等[27] 提出知识、能力、人格三方面的创新人才培育模式, 结合 教、学及课程考核方式改革展开论述。

\section{5. 实验环境建设与管理}

实验环境建设与管理方面研究包括实验平台研发设 计以及对实验室的建设管理。其中, 实验平台作为科学研 究的基地, 其重要性不言而喻。特别是对于工程问题而言, 在本科阶段, 它为学生提供了一个了解工程基本原理的机 会; 到了研究生阶段, 它作为进一步学习和解决实际问题 的工具更是不可或缺。而实验室作为实验活动的场所, 其 规范化建设与管理是学生正常参与实验教学的一大保障, 也是促进实验教学研究的突破口之一。

\subsection{1. 实验平台研发设计角度}

国内相关学科研究集中在工学, 主要关注实验平台的 建设与设计的思路、方法、途径、目标、内容, 以及实验 实施、考核与评价、运行效果与效益。包括基于 $\mathrm{Web}$ 的远 程实验室、基于浪潮集群系统的天气预报模式 (WRF) 实 验环境、PLC综合实训平台、电能质量综合实验平台、部 队院校电气工程研究生实验平台、基于真实实验对象的开 放电工电子网络实验系统、基于工程项目的控制实验平台、 机器人实验教学平台软件、机械工程学科的应用型研究生 实践教学平台、研究生专业综合实验平台、基于科研项目 的数控加工制造过程监测和控制实验平台、基于现场可编 程的通信与信息系统专业开放式研究生创新实验平台、面 向化学工程与技术学科研究生的创新实验项目设计、基于 气流床气化的煤质评价实验平台。

\subsection{2. 实验室建设角度}

针对非具体学科的研究有: 西北工业大学研究生创新 实验中心建设, 依托于实验教学示范中心的研究生创新实 践基地, 搭建学术攻关、实验实践、校企合作和校园文化 等研究生自主创新的平台、公共教学科研实验平台, 中国 科学技术大学以需求为导向的面向科研和教学的公共实 验中心。从具体学科角度, 医学方面有头颈肿瘤分子生物 学实验平台、医学形态学实验技术平台。工学方面有机械 工程学科研究生综合实验中心、物理学研究生创新实验平 台、光电技术研究生综合实验中心、光电子技术专业高层 次人才培养实验基地、电力电子技术实践创新平台、面向 矿业工程的力学实验示范基地、校企共建符合材料实验平
台。管理学方面有会计专业硕士(MPAcc)虚拟仿真教学实 验中心、研究生财务实验教学基地。

\subsection{3. 实验室管理角度}

研究包括网络化虚拟电子仪器库、新加坡国立大学的 实验室管理模式、院级大实验室共享管理模式、教学科研 实验室开放与资源共享、研究生参与实验室管理、高校科 研实验室安全管理、北京师范大学微生物学实验室模块化 管理实践、研究生创新实验评价、实验室药品仪器管理系 统开发等。

这些有关实验环境的研究大多结合了先进的计算机 技术、专业技术或者开放的管理思想等，因此能够从较新 颖的角度对于实验环境的建设给出指导性观点, 值得进行 匹配分析与借鉴。

\section{3. 文献分析与研究展望}

研究生实验教学作为高等学校教育的一项重要内容, 是培养高素养专业人才的一个关键环节。现有的课程级实 验研究、专业级实验研究、学科或学院级公共实验研究、 跨学科创新实验及实验环境建设与管理等方面实验教学 研究将为高校开展研究生实验教学活动提供科学的依据。 根据文献研究现状, 研究生实验教学的相关研究进展可总 结如下:

各类研究成果不均。从现有文献数量上来看, 根据以 上研究生实验教学研究的分类, 我国学者的研究成果集中 在课程级实验以及实验环境建设与管理两方面。这说明现 有相关研究与高校所设课程的依赖性较强, 同时国内对于 实验教学环境相当重视。

学科分布失衡。现有研究成果所涉学科种类较为广泛, 主要包括工学、生物学、医学、管理学等。其中对于工学 的相关研究最为普遍, 而生物学相关研究亦占据较大比例。 目前来看, 由于相关研究问题几乎各不相同, 研究成果广 而不深, 暂未形成较为平衡的、结构清晰的实验教学结构。

差异化难以协调。大多学者依据所在高校总体实验教 学情况或特色学科建设进行研究分析, 由于各高校教学政 策、教学水平、师资力量、资金投入等方面差异较大, 所 得研究成果难以直接借鉴, 这些因素共同制约了国内高校 研究生实验教学的发展。其次, 水平相近的高校间缺乏相 互合作, 交流沟通较少, 高校专家凭借各自经验摸索教学 手段, 难以实现较大进步。

综合我国研究生实验教学现状与上述分析, 可进一步 深化的研究方向如下:

研究生实践能力培养的体系化研究。将研究生实验教 学中的课程实验、专业实验、学科级实验甚至跨学科实验 等配合、衔接, 构成一个系统。从全局出发, 抓住整体和 要害, 灵活有效的处理学生培养过程中存在的问题。当然, 这方面研究可能需要与学科、学院甚至学校整体情况结合, 进而具体化细节方面。由于思维高度与实践要求较为苛刻, 目前暂未发现相关研究。随着高校实验教学活动的进一步 推进, 期待未来对于该方面的研究有所进展。

研究生公共实验教学研究。目前已知多所院校开展了 公共实验教学, 但对于公共实验方面的教学研究成果仍然 
较少, 有待加强。特别是针对具体学科或学院的公共实验 教学研究, 学者们可从公共实验的教学内容、形式、环境、 学分、定位等多方面论述各自教学经验, 以供各院校通过 信息计量分析对各自的公共实验内容进行调整与改进。

科技化实验教学研究。作为研究生的科研重要场所, 实验室或实验平台的建设可为推进人才孵化, 培养有实力 做实在事的接班人奠定基础。随着信息技术的发展, 诸多 $\mathrm{O} 2 \mathrm{O}$ 模式的实验环境建设如远程虚拟结合物理实验室等, 可以将信息技术手段渗透到高校的实验教学活动中, 改善 实验教学管理、反馈和评估体系, 解决部分实验的环境问 题和可持续性需求。对于大型仪器设备或各类创新实验的 虚拟化场所提供, 更能避免昂贵的实验室设备, 对实验教 学起到一定促进作用。但是由于各高校水平与资金投入等 因素限制, 相关建设的普及化程度较低, 学者们应重视已 有案例, 进一步思考与完善信息化实验环境建设方案, 得 出更多现实可行的研究生实验教学成果。

\section{致谢}

本文为合肥工业大学2018年研究生培养质量工程项 目(2018YJG01)的阶段性成果之一。

\section{参考文献}

[1] 教育部.教育部、国家发展改革委、财政部关于深化研究生 教育改革的意见 [EB/OL]. [2013-04-19].http://www.moe.gov.cn/srcsite/A22/s7065/2013 04/t20130419_154118.html.

[2] 范冬梅, 潘信吉, 胡今鸿. 从创新能力的培养谈研究生实验教 育的改革 [J].实验室研究与探索,2008,27(8):7-9。

[3] 范冬梅.从教育三元素论研究生实验教育改革 $[\mathrm{J}]$. 实验室研 究与探索,2009,28(7):108-109。

[4] 张洋, 芮国胜, 张嵩,王瑞, 张海波.军队院校研究生实验教学 分析与发展研究[J]. 实验技术与管理,2017,34(s1):125-127。

[5] 张培玉,曲洋,于德爽,李悦,孙文寿. 环境工程硕士实验与实 践环节的改革与实践 [J]. 实验技术与管 理,2010,27(6):137-139。

[6] 李彦文,张娜, 尹华,莫测辉,薛枫.环境专业硕士研究生实验 教学探索[J].实验技术与管理,2010,27(7):148-150。

[7] 秦春节,陈琍,王成,鲁建厦,陈勇.基于工程实践的物流工程 专业实验教学 $[J]$. 实验室研究与探索,2016,35(1):179-182。

[8] 娄敏,陈建民. 具有海洋石油特色的船舶与海洋工程专业硕 士实践教学体系建设 [J].船海工程,2016,45(6):141-143。

[9] 章牧,赵新建,罗靖.旅游管理实验教学中心创新性教学体系 [J].实验室研究与探索,2009,28(6):230-234。

[10] 赵新建,张小红,章牧.面向本科与研究生协同的衔接性实验 教学体系 [J].实验室研究与探索,2011,30(8):395-399。
[11] 钱猛,杨娜,陈军,沈振国.微生物学类研究生创新技能培养体 系的探索——南京农业大学为例 [J]. 微生物学通 报,2016,43(4):839-844。

[12] 郑晓东,刘向东,刘旭,毛小兰,闻春敖.知识、能力、素质并重 —光学工程研究生实验教学新模式的探索 [J]. 光学技 术,2008,34(s1):309-311。

[13] 程林松,李春兰.油气田开发工程专业研究生实验教学培养 模式探讨 [J].实验室研究与探索,2011,30(6):322-324。

[14] 张英,郝林琳,李占军,李莉,任林柱,欧阳红生,逢大欣,张丽颖. 动物学研究生实验教学体系的构建与思考 [J]. 黑龙江畜牧 兽医,2018(17):219-221。

[15] 郑永杰,田景芝,纳丽娜, 荆涛,陈国良,王丽艳.日本工科研究 生实践教学的经验及其启示 [J].教育探索,2011(6):153-155。

[16] 刘丽琳,孙久厚.建立公共实验平台培养研究生创新能力 $[\mathrm{J}]$. 学位与研究生教育,2002(7):56-58。

[17] 郑冬梅,王悦.构建研究生实验教学体系,培养研究生创新能 力 $[\mathrm{J}]$.实验技术与管理,2010,27(5):146-147。

[18] 张建宇,李羽让,孙东平,汪信. 以学科实验平台为基础构建研 究生公共实验教学体系的构想与实践 $[\mathrm{J}]$. 学位与研究生教 育,2012(3):33-35。

[19] 林小虎,杜涁,杨立铭,宋爱君,郭振清,宋小飞.农科类研究生 实验教学体系改革探索与思考 $[\mathrm{J}]$. 安徽农业科 学,2010,38(18):9872-9873。

[20] 赵琴,江启峰,刘小兵.依托重点实验室构建地方高校研究生 实验教学体系 $[J]$.实验技术与管理,2015,32(12):22-25。

[21] 孙腊珍,张增明,叶邦角,宋克柱,许立新.科研能力培养和研 究生实验教学体系的构建 [J]. 学位与研究生教 育,2010(6):58-60。

[22] 间连山,潘炜,罗斌,邹喜华,张志勇.结合前沿实验研究提高 研究生培养质量的探索 [J]. 实验技术与管理,2011,28(3):5-6。

[23] 杨晓华,吴小健,郑建武,张华峰,肖宁,赖江华.开展自主创新 实验培养创新型医药专业人才 $[\mathrm{J}]$. 实验室研究与探 索,2015,34(5):155-158。

[24] 武卫莉,刘喜军,贾宏葛,佟丽,程伟东.高分子材料工程硕士 创新实验能力培养模式研究 [J]. 高分子通报,2016(1):94-97。

[25] 刘华.借鉴国外经验改革研究生教学模式 [J].实验技术与管 理,2017,34(05):158-160。

[26] 杨晓红,王凯渊,王兆春, 唐颖,吴嘉荟. 设计性实验教学在研 究生创新能力培养中的探索与实践 [J].西南师范大学学报 (自然科学版),2018,43(11):166-171。

[27] 孙建和, 王恒安, 马婧姣, 严亚贤. 以培养研究生创新思维为目 标的 “兽医分子病毒学” 教学模式的探索与实践 [J].微生物 学通报,2018,45(11):2517-2522。 